\title{
Financiación con gradientes a sectores no aptos para credito bancario analizados con Sotfware GEOGEBRA'
}

\author{
Gradients finance sectors not for bank credit sotfware discussed with GEOGEBRA
}

\author{
Jorge Iván Jiménez Sánchez²
}

\section{FORMA DE CITACIÓN}

Jiménez, J. I. (2013). Financiación con gradientes a sectores no aptos para crédito bancario analizados con software GEOGEBRA. Revista Dimensión Empresarial, vol. 11, núm. 2, pp. 51-61

\section{RESUMEN}

El artículo detalla el uso de herramientas computacionales que pueden ser utilizadas por la gerencia y la banca en el análisis de alternativas de crédito y en la búsqueda de nuevos mecanismos de acceso a la financiación que acá se expone y que pueden ser utilizados en la búsqueda de productos bancarios. Los gradientes son formas de pago diferentes a las tradicionales en el que las cuotas varían en cada periodo. El uso de gradiente mejora el flujo de caja, acá los pagos no son iguales si no que aumenta o disminuyen periódicamente según la necesidad. GEOGEBRA es un software que permite interactuar con los elementos que componen un crédito, su interfaz cuenta con un sistema de geometría dinámica para el análisis, la visualización y estimación de todo tipo de cálculos, es una herramienta básica para la toma de decisiones.

Palabras Clave: gradiente aritmético, sector financiero, GEOGEBRA, análisis de crédito, formas de pago.

\section{ABSTRACT.}

The article details the use of computational tools that can be used by management and banking in the credit analysis of alternatives and the search for new mechanisms of access to finance that here is exposed and can be used in the search for banking products. The gradient payment methods are different from the traditional on which fees vary each period. The use of gradient improves cash flow, payments here are not the same if not increase or decrease as needed periodically. GEOGEBRA is a software that allows you to interact with the elements of a credit, its interface has a dynamic geometry system for analysis, visualization and estimation of all kinds of calculations, is a basic tool for decision-making.

Keywords: arithmetic gradient, financial sector, GEOGEBRA, credit analysis, financial payment.

\section{INTRODUCCIÓN.}

A pesar de los avances logrados en materia de bancarización, no se aplican nuevas formas de pago que faciliten el acceso al crédito a "sectores no aptos", definidos así por ASOBANCARIA. Esto se refleja en que las formas tradicionales de pago y el cubrimiento de las necesidades sigue siendo muy bajo. Se dice que en Colombia el nivel de bancarización está al alcance de la casi totalidad de los sectores, pero a pesar de esta condición, el acceso al crédito solo llega a 12 de cada 100 de las empresas (Portafolio, 2013), mucho más difícil es el acceso al crédito para sectores cuyos flujos de caja tienen que dar espera, como lo son el de la construcción o el sector de la agricultura y municipios (Obras). Por esta razón, se requiere que la banca cuente con nuevas modalidades y nuevos métodos de pago que ayuden y permitan el avance económico.

\footnotetext{
Artículo de Investigación. Recibido en septiembre 15 de 2013. Aceptado en noviembre 2 de 2013. Este artículo hace parte de la ponencia presentada en el IX congreso internacional de matemáticas, EIMAT organizado por la Universidad del Atlántico en la ciudad de Barranquilla, Colombia titulada "cálculo de la cuota para préstamos con "Gradiente" aritmético creciente y su aplicación en software matemático interactivo, como herramienta de apoyo empresarial y pedagógica" y la cual es producto del proyecto de investigación: Canales y Mecanismos de Acceso a la Financiación, Fondeo y Préstamos a Sectores No Aptos para el Sistema Financiero Colombiano.

2 Administrador de Empresas de la Universidad Cooperativa de Colombia U.C.C., Colombia; Especialista en Gestión Financiera Empresarial Universidad. de Medellín, Colombia; Magíster en Administración de Empresas con especialidad en finanzas corporativas, Universidad Viña del Mar, Chile; Certificación Internacional "Master Financial Profesional (MFP)" de la American Academy of Financial Management (AAFM),EE.UU; Docente tiempo completo de la Institución Universitaria, Tecnológico de Antioquia, Medellín, Colombia. Correo: jijs294@gmail.com.
} 
Este artículo explica como el uso de gradientes apoya la búsqueda de modalidades de pago diferentes a las tradicionales ya que poseen muchas bondades a la hora de formalizar un crédito. Utilizar las matemáticas financieras junto con herramientas computacionales sirve de apoyo y complementa la toma de decisiones para la gerencia al igual que para la banca al momento de solicitar $u$ otorgar un préstamo (José \& Valenzuela, 2001), también ayuda a las partes a comprender el comportamiento de estas formas de pago.

Los gradientes surgen como forma de pago y apoyo a la búsqueda de nuevos canales y mecanismos de acceso a la financiación, en especial para esos sectores, las modalidades de pago diferentes a las tradicionales pueden utilizarse ya que beneficiaría a los usuarios de crédito de manera notable. El uso y la comprensión de otras formas de pago favorece a la comunidad en su conjunto, los cuales forman parte de la infraestructura productiva a través de la cual se moviliza el dinero en una economía, permitiendo que los clientes de un banco efectúen pagos con condiciones diferentes que se adapten a las partes y con lo cual se dinamicen las transacciones.

En matemáticas financieras los gradientes son anualidades o serie de pagos periódicos, en los cuales cada pago de cada cuota es igual al anterior más una cantidad. Estos son los llamados gradientes crecientes, pero también pueden presentarse pagos de forma decreciente o de forma escalonada (Meza, 2008).

La primera parte de este articulo analiza lo relacionado con los gradientes y sus formas, la segunda parte del articulo relata cómo se pueden aplicar con el software geométrico GEOGEBRA (Hohenwarte, 2013) y el análisis de un crédito, y como este aporta a la toma de decisiones. El programa permite el montaje de todo tipo de fórmulas las cuales pueden graficarse en un plano cartesiano e interactuar con la hoja de Excel que contiene el programa. También se puede analizar el componente matemático de la fórmula en la ventana algebraica el cual nos ayuda en la comprensión de los elementos que la componen.

\section{METODOLOGÍA}

\section{El cálculo de una formula gradiente aritmético o lineal como apoyo a la toma de decisiones en la gerencia}

La matemática aplicada se refiere a todos aquellos métodos y herramientas matemáticas que pueden ser utilizados en el análisis o solución de problemas del área de las ciencias aplicadas o sociales (José \& Valenzuela, 2001). Las matemáticas financieras son una rama de las matemáticas aplicadas, enfocadas a la solución de problemas sobre el valor del dinero en el tiempo: monto, tasa de interés, anualidades, gradientes (pagos crecientes y decrecientes), etcétera.
La matemática financiera ofrece muchas modalidades de pago, el utilizarlas favorecería y fomentaría la bancarización, posibilitando la creación de nuevos productos y servicios financieros para estos sectores, beneficiando tanto a los usuarios como a las instituciones financieras. Para un buen funcionamiento de los mercados financieros y de la economía en general, es imprescindible contar con variados sistemas de pagos.

Los sistemas de pagos pueden estar expuestos a diversos riesgos, como son los riesgos financieros. Por ejemplo, un participante podría efectuar tardíamente un pago (riesgo de liquidez), o no poder pagar (riesgo de crédito) (Medina, 2008), afectando adversamente así al participante beneficiario o receptor de fondos. Al utilizar los gradientes ambas partes pueden pactar una mejor modalidad de pago, un ejemplo claro es el dinero solicitado para construcción, el sector agrícola o para una pyme, incluso para Municipios, que requieren obras inmediatas, en estos sectores el dinero invertido no puede empezar a fluir rápidamente y es acá donde otras formas de pago podrían ayudar a reducir el riesgo.

Para el análisis de este tipo de créditos es ideal contar con las herramientas computacionales como el Excel y en este caso el GEOGEBRA (GEOGEBRA, 2013), son un apoyo muy importante a la interpretación gráfica y matemática en busca del mejor de los resultados cuya comprensión y funcionamiento de este tipo de créditos permite modelar o visualizar problemas o situaciones matemáticas (Ferragina y otros, 2012). Pueden ser utilizadas como herramientas de apoyo a la gerencia y la banca en el análisis de un crédito ya que permite interactuar y efectuar cambios con el fin de apreciar cómo pueden variar las condiciones de un crédito, ayuda a la gerencia a determinar las acciones que se deben tomar con la finalidad de lograr cierto objetivo financiero.

Muchas empresas buscan endeudarse para poder impulsar el desarrollo y crecimiento empresarial. Para las empresas el apalancamiento (Gitman \& Núñez, 2003) es aprovechar la deuda para crecer, la fórmula que se expone en este artículo trae un resultado que puede definirse como gradiente a una serie de flujos de caja periódicos (José \& Valenzuela, 2001), los cuales poseen una ley de formación, refiriéndose a que estos flujos se pueden incrementar o disminuir con base en el flujo de caja anterior, por lo cual, sería ideal para la banca ofrecer estas modalidades de crédito.

Para que exista gradiente la serie de pagos debe cumplir con las siguientes condiciones: I) El número de pagos es igual al número de períodos. II) Los pagos se efectúan a iguales intervalos de tiempo. III) Todos los pagos cumplen con una ley de formación y, IV) Todos los pagos se trasladan al principio o al final a la misma tasa de interés (Morales, 2012).

Hay varios tipos de gradientes son I) El gradiente aritmético, el cual es un conjunto definido por una serie de pagos 
periódicos en la cual cada pago es igual al del periodo inmediatamente anterior, incrementado o disminuyendo en una cantidad de dinero constante. II) El gradiente geométrico, el cual es definido por una serie de pagos periódicos, en los cuales, cada pago es igual al del período inmediatamente anterior incrementado o disminuido en un mismo porcentaje, es decir aumentan o disminuyen en un valor variable, pero conservando el mismo porcentaje y, III) El gradiente escalonado los cuales son valores constantes durante los periodos de un año, pero que aumenta o disminuye en cada escalón en una cantidad fija de dinero o en una tasa constante (Guzmán, 2006).

Es de anotar que los gradientes pertenecen a las series variables de amortización de cuotas (Aguirre, 2008), pero con la diferencia que cada periodo de pago varía de manera diferente a las uniformes donde las cuotas son iguales, las series gradiente pueden ser anticipadas o vencidas y pueden ser crecientes o decrecientes. Los gradientes crecientes son muy ideales para los gerentes que planean pagar un crédito con cuotas muy bajas al principio, lo cual sería ideal para muchas sectores ya mencionados porque las cuotas se van incrementando en cada período de pago y van sumando a la cuota el valor del gradiente (Serna \& Rojas, 2012), como se muestra en el Gráfico № 1.

Ahora la característica de los gradientes decrecientes (Navarro, 2013), la constituye el que al iniciar las cuotas son relativamente altas (más altas que las cuotas de la serie uniforme) y van disminuyendo en un porcentaje equivalente del valor del gradiente. El monto de los intereses pagados es proporcionalmente bajo, la amortización a capital se realiza más rápido. Este tipo de créditos es ideal cuando los gerentes tienen como fin pagar las deudas abonando a capital lo más pronto posible, es otra alternativa al crédito el cual la gerencia debe analizar sobre cuál es más conveniente o no (Horne \& Wachowics, 2002).

Los gradientes escalonados (Morales, 2012), tienen como característica fundamental la variación entre grupos de cuotas, en la cual un grupo de cuotas constituyen una serie uniforme y periódicamente varían en una cantidad en pesos, en el caso de la serie aritmética, o también porcentual si es el caso, igualmente se pueden presentar de manera creciente o decreciente por grupos de cuotas, su representación geométrica se puede ver en el gráfico $\mathrm{N}^{\circ} 2$.

Gráfico Nº1. "Gradiente" creciente y decreciente"
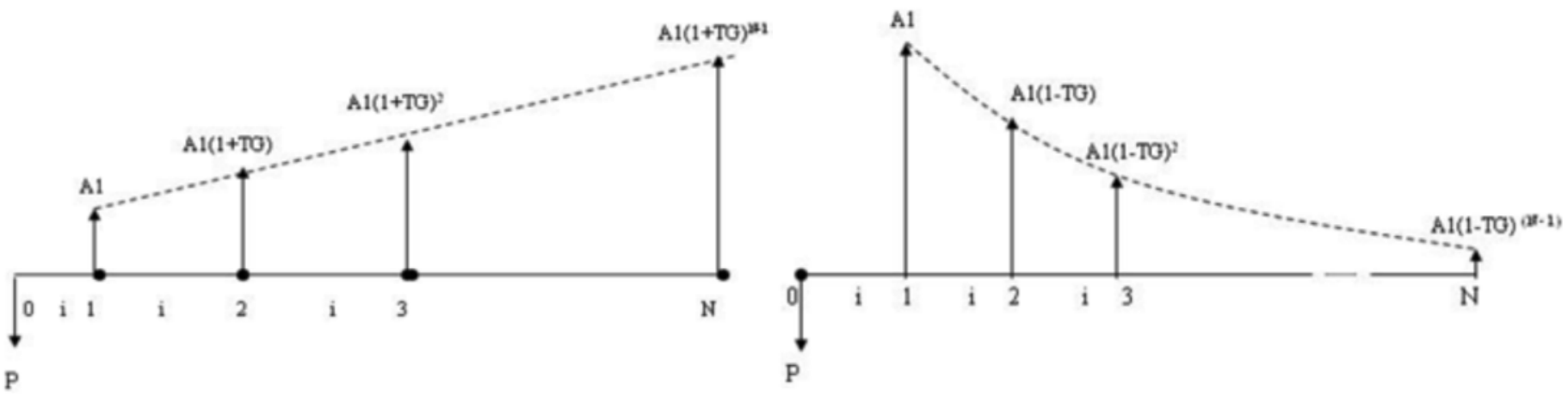

Fuente: Navarro (2013).

Gráfico $\mathbf{N}^{0} 2$. Gradiente escalonado creciente.

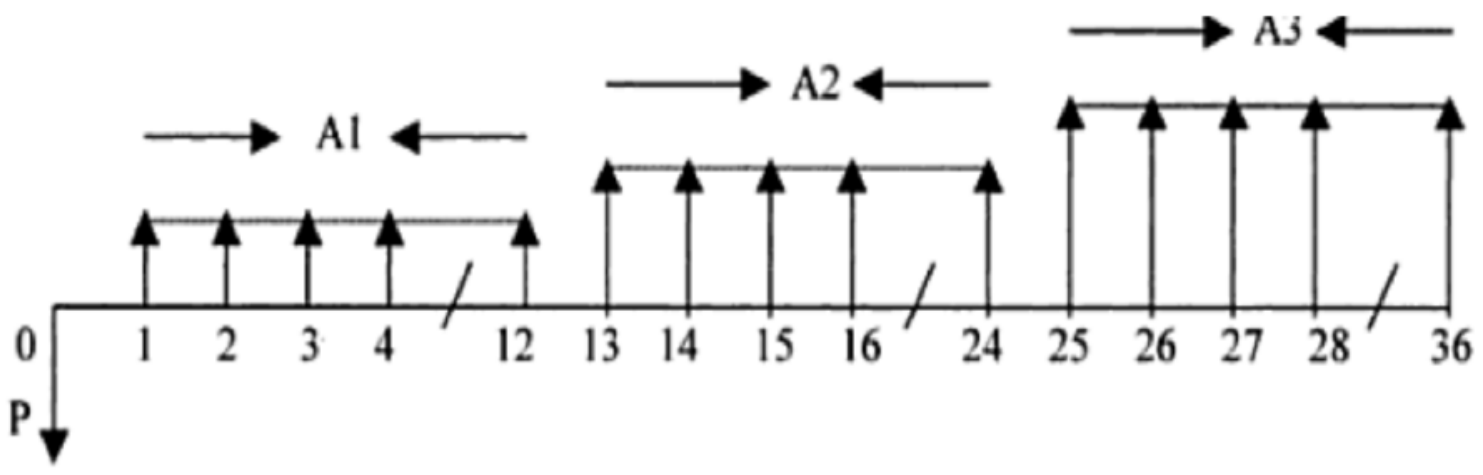

Fuente: Meza $(2008,411)$ 
Cabe anotar que el mejor plan de amortización es el que se ajuste a la capacidad de pago y que compense con los sus futuros ingresos. Un sistema de amortización estipula como se va realizar el pago del préstamo, en este caso en cuotas mensuales con gradiente que incluye capital, intereses y que se cancelan durante el plazo pactado, estos se diferencian por la velocidad con que se paga el capital adeudado. Colombia requiere de implantar nuevas formas y modalidades de crédito diferentes a las cuotas iguales y constantes para ofrecerlos a los sectores que más lo requieren y que son impulsadores y dinamizadores de la economía.

\section{Descripción del Modelo matemático}

Para la descripción del uso de la herramienta computacional utilizaremos la fórmula matemática gradiente creciente aritmético vencido en el cual se considera que los pagos en cada periodo serán diferentes. Estos se identificaran con un subíndice que indica el consecutivo del pago. De acuerdo con la ley de formación, en este caso, cada pago será igual al anterior más una constante, así como se muestra a continuación en la tabla № 1 .

Tabla №1. Cuota pagos con gradiente.

$$
\begin{aligned}
& A 1=\text { Primer pago } \\
& A 2=A 1+G \text { Segundo pago } \\
& A 3=A 2+G=A 1+2 G \text { Tercer pago } \\
& A 4=A 3+G=A 1+3 G \text { Cuarto pago } \\
& A n=A 1+(n-1) G \text { Nuevo pago }
\end{aligned}
$$

Fuente: diseño propio basado en Meza (2008).

Los gradientes, como ya se observó, pueden ser aritméticos, geométricos o escalonados, crecientes o decrecientes, pueden calcularse a través de cuotas anticipadas o vencidas, igualmente pueden calcularse con base en conocer el valor presente o el valor futuro (Salcedo, 2002). De ello resultan muchas formas y combinaciones para el cálculo de los gradientes.

El modelo matemático que se representa en este trabajo considera una operación en la cual un préstamo se paga en una serie de cuotas formada a través de un gradiente aritmético creciente y vencido y a una tasa de interés efectiva por periodo, durante $\mathrm{n}$ periodos. La situación se muestra: Analizando el segundo flujo del gráfico $\mathrm{N}^{\circ} 1$, se observa que el incremento de la cuota gradiente $(G)$ comienza en el periodo $\mathrm{N}^{\circ} 2$. Para hallar el valor de la cuota necesitamos conocer datos como el valor presente del crédito, el gradiente en pesos, la tasa de interés y el tiempo en el cual se pagara el crédito. Con la siguiente formula se halla la cuota A o sea la cuota $\mathrm{N}^{0} 1$ gradiente (José \& Valenzuela, 2001). Ver Gráfico N³.
Gráfico $\mathbf{N}^{\circ} 3$. Formula para el cálculo de un gradiente creciente aritmético vencido

$$
A=\left[\frac{\mathrm{P}-\left[\frac{G}{i}\left[\frac{(1+i)^{n}-1}{i(1+i)^{n}}\right]-\left[\frac{n}{(1+i)^{n}}\right]\right]}{\left[\frac{(1+i)^{n}-1}{i(1+i)^{n}}\right]}\right]
$$

Conocido el valor de la cuota $N^{0} 1$ Se podrá calcular el valor de las siguientes cuotas en cada periodo, Agregamos a cuota $\mathrm{A}$ a la siguiente formula:

$$
\text { Cuota } n^{0} \ldots \ldots . . .=A+(\text { Cuota- } 1) G \text {. }
$$

Tabla №2. Elementos que componen de la formula gradiente aritmético creciente vencido.

$$
\begin{aligned}
& \mathrm{P}=\text { Valor presente de la serie de "Gradientes } \\
& \mathrm{A}=\text { Valor de la primera cuota de la serie } \\
& \mathrm{i}=\text { Tasa de interés de la operación } \\
& \mathrm{n}=\text { número de pagos o ingresos } \\
& \mathrm{G}=\text { "Gradiente" }
\end{aligned}
$$

Fuente: Meza (2008)

O lo que es igual (Meza, 2008)

$$
A=G\left(\frac{1}{i}\right)\left[1-\frac{n i}{(i+1)^{n}-1}\right]
$$

Donde la anualidad A, dado un gradiente $\mathrm{G}$ está dada por:

$$
A=G\left[\frac{1}{i}-\frac{n}{(i+1)^{n}-1}\right]
$$

\section{Descripción del software GEOGEBRA}

Es importante contar con software para la comprensión del comportamiento de un gradiente, en este caso se trae a consideración el GEOGEBRA, elaborado por Hohenwarter \& Hohenwarter (2008) para la enseñanza de las matemáticas. GEOGEBRA puede ser usado en física, cálculo, álgebra, así como todo tipo de proyecciones comerciales y financieras. Es una herramienta que para ser usada en el campo estratégico, este trabajo muestra el cálculo de la formula gradiente aritmético o lineal creciente vencido, aplicada para el análisis de un crédito (Meza, 2008) mediante la representación gráfica, para lo cual se diseña un OVA ${ }^{3}$

3 OBJETO VIRTUAL DE APRENDIZAJE (OVA): hace referencia a todos los materiales audiovisuales estructurados de una manera significativa, los cuales tienen un propósito educativo y corresponden a un recurso de índole digital que puede ser distribuido en medio magnético y/o consultado en el aula virtual. Algunas muestras de ovas pueden ser las animaciones, videos, audios, simuladores, entre otras. 
(Colombia Aprende, 2013), en la cual se puede observar y analizar el comportamiento de este tipo de crédito.

En los últimos años, GEOGEBRA, ha mostrado ser un apoyo muy importante para todo de aplicaciones de cálculo con matemáticas y, también, por los interesados en incorporar herramientas tecnológicas en su trabajo. El programa permite realizar construcciones con puntos, vectores, segmentos, rectas y secciones cónicas, el software cuenta con tres vistas, ver Gráfico № 4.

GEOGEBRA ofrece tres perspectivas diferentes de cada objeto matemático (Costa \& Vacchino, 2013): una vista gráfica, una numérica y una vista algebraica que permite incorporar funciones. Además, una vista de hoja de cálculo (Similar a Excel). Esta multiplicidad permite apreciar los objetos matemáticos en tres representaciones diferentes: gráfica (como en el caso de puntos, gráficos de funciones), algebraica (como coordenadas de puntos, ecuaciones) y en celdas con datos en una hoja de cálculo. Cada representación del mismo objeto se vincula dinámicamente a las demás en una adaptación automática y recíproca que asimila los cambios producidos en cualquiera de ellas, más allá de cuál fuera la que lo creara originalmente, (Matematica, 2012).

\section{RESULTADOS Y DISCUSIÓN}

\section{Calculo aplicando la fórmula del gradiente aritmético creciente vencido con el GEOGEBRA}

El pago con gradientes ayuda a disminuir el riesgo de crédito, las modalidades de pago son variadas y permiten planear la mejor forma de cancelar una deuda. La buena selección de un crédito de acuerdo con estudios relacionados con el tema ha demostrado que incrementa la productividad en las empresas. Pero el crédito en si es solo una herramienta, es necesario concientizar a los bancos para que incorporen mejores servicios y variadas modalidades de pago y los ofrezcan a los sectores no aptos para crédito, si se quieren lograr niveles más eficientes de bancarización y, por ende, de desarrollo económico y bienestar social.

Con las formulas definidas y comprendiendo el concepto del gradiente aritmético creciente vencido se puede observar el resultado en el análisis de un crédito, a través del planteamiento de un ejercicio y su respectiva solución: la Compañía XYZ necesita un crédito de $\$ 13.500 .000$ millones de pesos y recurre al banco $A$. Allí le proponen pagar el crédito en 12 meses a una tasa del $3 \%$ mensual. Si se tiene como condición un gradiente creciente vencido de $\$ 150.000$ pesos cada mes, ¿cuál será el valor de cada cuota con gradiente"?

Las definiciones de variables se presentan en la tabla $\mathrm{N}^{0} 3$.

Tabla №3. Datos para hallar la cuota de pagos con gradiente creciente, alternativa del banco $\mathrm{A}$

$$
\begin{array}{|l|l|}
\hline \mathrm{n}=\text { tiempo en meses. } & \mathrm{n}=12 \\
\mathrm{i}=\text { la tasa de interés. } & \mathrm{i}=3 \% \text { mensual } \\
\mathrm{P}=\text { capital solicitado. } & \mathrm{P}=\$ 13.500 .000 \\
\mathrm{G}=\text { gradiente } & \mathrm{G}=\$ 150.000 \\
\mathrm{~A}=\text { cuota a pagar } & \mathrm{A}=\text { cuota a pagar } \\
\hline
\end{array}
$$

Fuente: autor con base en Orozco (2008).

Gráfico $N^{\circ} 4$. Vistas del programa GEOGEBRA

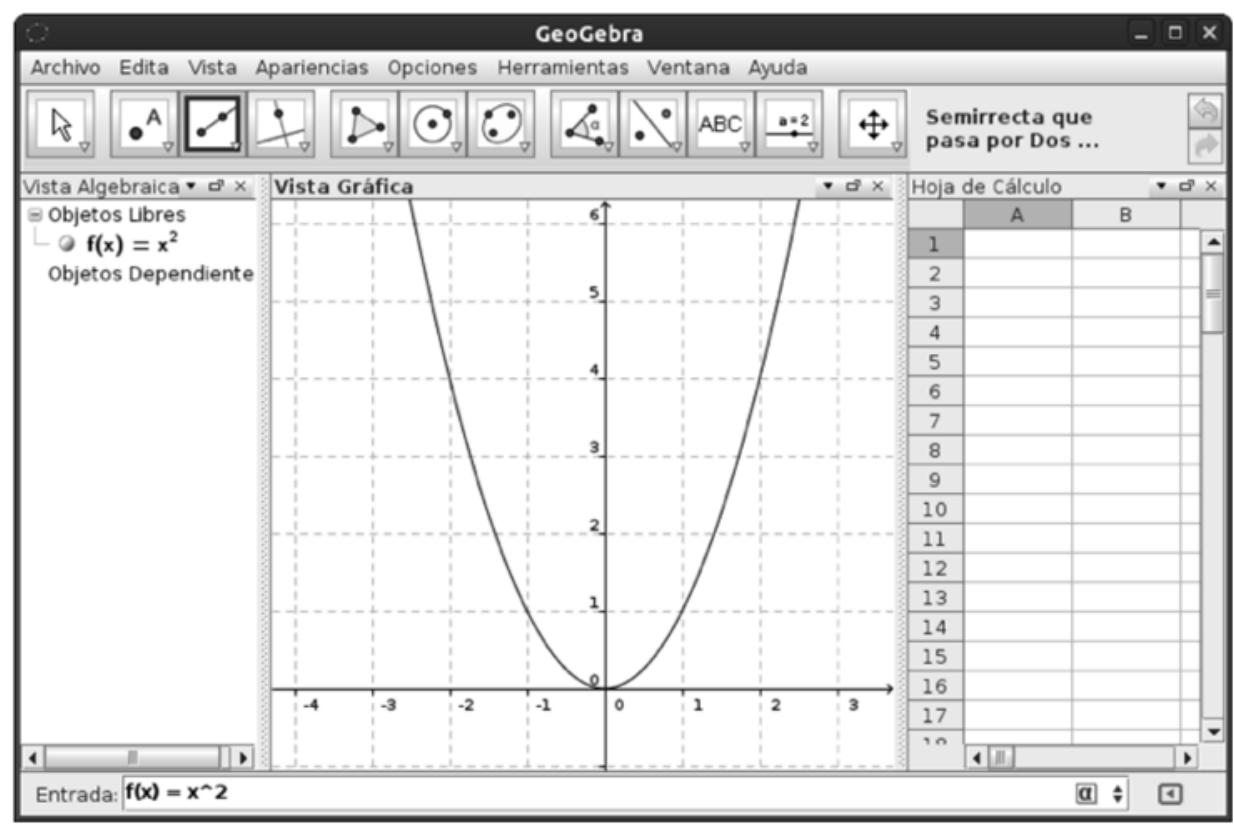

Fuente: Matematica, 2012. 
Los resultados del cálculo aplicando el software de análisis computacional GEOGEBRA, como ya se observó el programa cuenta con tres vistas, que permiten el análisis del crédito, a continuación se puede apreciar la aplicación y sus resultados en una hoja de cálculo del programa.

\section{Calculo de la tabla de amortización con gradiente cre- ciente vencido en la Hoja de cálculo del GEOGEBRA}

El programa cuenta con una hoja de Excel en la que se puede incorporar la fórmula de gradiente aritmético lineal vencido y hallar la solución. Se elabora la tabla de amortización para hallar el valor de la cuota en cada uno de los periodos, de acuerdo con las condiciones el préstamo deberá pagarse en 12 cuotas cada una varia con respecto a la anterior en $\$ 150.000$ pesos, el cálculo del primer gradiente se realiza con la formula incorporada a la celda $\mathrm{D} 10=(\mathrm{B} 1-\mathrm{B} 3 / \mathrm{B} 4$ $\left.\left(\left((1+B 4)^{\wedge}(B 2)-1\right) /\left(B 4(1+B 4)^{\wedge}(B 2)\right)-B 2 /(1+B 4)^{\wedge}(B 2)\right)\right)$ $/\left(\left((1+B 4)^{\wedge}(B 2)-1\right) /\left(B 4(1+B 4)^{\wedge}(B 2)\right)\right)+(A 10-1) B 3 y$ se calcula la fórmula para los siguientes periodos en cada una de las celdas, ver gráfico $\mathrm{N}^{\circ} 5$.

Se puede apreciar el comportamiento del gradiente creciente vencido, en el que la primer cuota corresponde a $\$ 583.960$ y va creciendo en cada periodo hasta encontrar que el último pago corresponde $\$ 2.233 .960$, para el sector construcción, agrícola, pymes y municipios este este tipo de créditos serían muy beneficiosos ya que darían un respiro a los sectores.

\section{Calculo con la formula gradiente propia de las mate- máticas financieras utilizando la vista algebraica del GEOGEBRA}

GEOGEBRA es muy dinámico y fácil de trabajar tomamos la fórmula de la celda D10 incorporada en Excel y con el comando propiedades definimos para el eje de las $x\left(\mathrm{~N}^{\circ}\right.$ cuota) con la función $\mathrm{Ag}(\mathrm{x})=\left(\mathrm{B} 1-\mathrm{B} 3 / \mathrm{B} 4\left(\left((1+\mathrm{B} 4)^{\wedge}(\mathrm{B} 2)\right.\right.\right.$ - 1) / (B4 $\left.\left.\left.(1+B 4)^{\wedge}(B 2)\right)-B 2 /(1+B 4)^{\wedge}(B 2)\right)\right) /(((1+$ $\left.\left.B 4)^{\wedge}(B 2)-1\right) /\left(B 4(1+B 4)^{\wedge}(B 2)\right)\right)+(x-1) B 3$, el programa automáticamente incorpora la formula con sus componentes, se puede apreciar en el la fórmula de gradiente creciente vencido. Ver Gráfico Nº6.

La vista algebraica nos muestra la estructura de la formula, la gerencia podrá analizar el comportamiento del crédito con gradiente creciente vencido en cada cuota y conocer el valor de cada una de ellas, se podrán efectuar cambios con el fin de determinar el resultado más óptimo y así tomar la decisión más adecuada.

Gráfico ํ⒌ Vista hoja de cálculo para el cálculo del gradiente creciente vencido

\begin{tabular}{|c|c|c|c|c|c|}
\hline his & 田血 & $\{1,2\}$ & \multicolumn{3}{|c|}{$\begin{array}{l}\text { Elige y Mueve } \\
\text { Arrastra o selecciona objetos (Esc) }\end{array}$} \\
\hline$=1$ & $\mathbf{N}$ & 拝 恶 & \multicolumn{2}{|l|}{ 明 - } & \\
\hline \multirow[t]{2}{*}{ D10 } & $\theta \approx$ & \multicolumn{4}{|c|}{$=\left(B 1-B 3 / B 4\left((C 1+B 4)^{n}(B 2)-1\right) /\left(B 4(1+B 4)^{n}(B 2)\right)-E\right.$} \\
\hline & A & $\mathbf{B}$ & c & $\mathbf{D}$ & $E$ \\
\hline 1 & $\mathbf{P}$ & 13500 & en miles & & \\
\hline 2 & $\mathbf{n}$ & 12 & & & \\
\hline 3 & $\mathbf{g}$ & \multirow{2}{*}{$\begin{array}{r}150 \\
0.03\end{array}$} & en miles & & \\
\hline 4 & i & & & & \\
\hline 6 & $A g(x)$ & 583.96 & primer cuota & & \\
\hline \multicolumn{6}{|l|}{7} \\
\hline 8 & PERIODO & CAPITAL & INTERESES & CUOTA & SALDO \\
\hline 9 & $\mathbf{0}$ & & & & 13500 \\
\hline 10 & 1 & 178.96 & 405 & 583.96 & 13321.04 \\
\hline 11 & 2 & 334.33 & 399.63 & 733.96 & 12986.7 \\
\hline 12 & 3 & 494.36 & 389.6 & 883.96 & 12492.34 \\
\hline 13 & 4 & 659.19 & 374.77 & 1033.96 & 11833.15 \\
\hline 14 & 5 & 828.97 & 354.99 & 1183.96 & 11004.18 \\
\hline 15 & 6 & 1003.84 & 330.13 & 1333.96 & 10000.34 \\
\hline 16 & 7 & 1183.95 & 300.01 & 1483.96 & 8816.39 \\
\hline 17 & 8 & 1369.47 & 264.49 & 1633.96 & 7446.92 \\
\hline 18 & 9 & 1560.56 & 223.41 & 1783.96 & 5886.36 \\
\hline 19 & 10 & 1757.37 & 176.59 & 1933.96 & 4128.99 \\
\hline 20 & 11 & 1960.09 & 123.87 & 2083.96 & 2168.9 \\
\hline 21 & 12 & 2168.9 & 65.07 & 2233.96 & $\mathbf{0}$ \\
\hline
\end{tabular}

Nota: cifras en miles de pesos.

Fuente: cálculos propios con GEÓGEBRA. 
Gráfico N6. Vista algebraica cálculo del gradiente escalonado creciente, realizado en GEOGEBRA

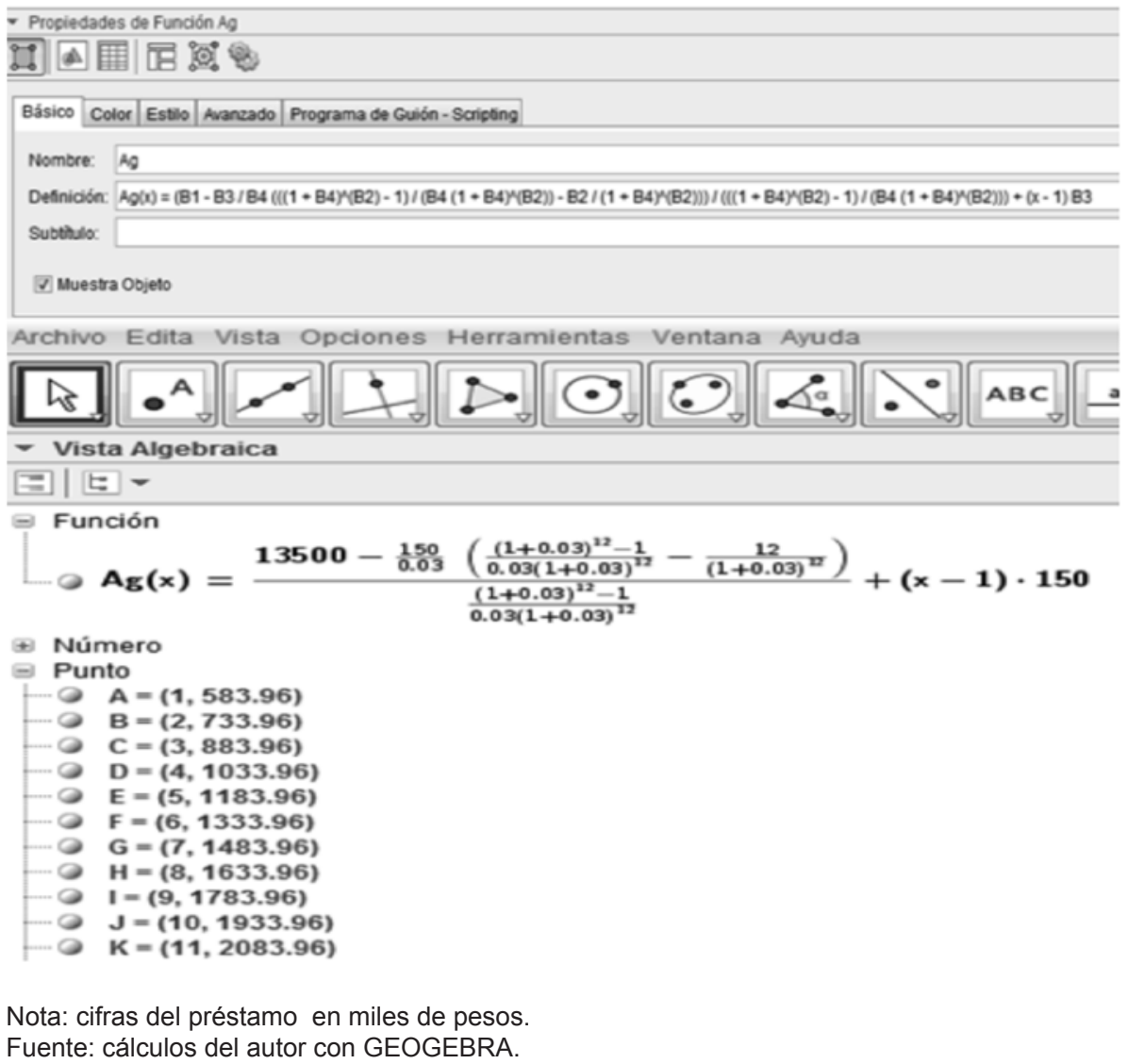

\section{Gráfico de amortización del gradiente creciente vencido utilizando la vista gráfica del "GEOGEBRA}

No menos dinámica e interactiva es la vista gráfica del programa GEOGEBRA, allí podemos observar representado en el plano cartesiano del programa el valor de cada una de las cuotas con gradiente creciente vencido. Se puede apreciar la función definida la cual se crea de manera automática con los menús y herramientas que ofrece el programa. Se pueden trazar puntos e interpretar en el plano cartesiano lo que deseamos analizar de una manera muy simple.

Sobre la recta trazada por la función $\mathrm{Ag}(\mathrm{x})$ se pueden apreciar el valor de cada pago; con ayuda del menú se definen los puntos y se crea un menú para ingresar datos, como la tasa el interés, el $N^{\circ}$ de cuotas, el capital solicitado etcétera. Gráficamente podemos apreciar cualquier cambio presentado, el programa ayuda en la comprensión sobre cómo funcionan este tipo de pagos y ayuda en la toma de decisiones.

Se puede observar que la cuota $\mathrm{N}^{0} 1$ tiene un valor de $\$$ 583.960 y la cuota $\mathrm{N}^{\circ} 12 \$ 2.233 .960$ a muchas empresas que inician proyectos a largo plazo y en la que sus ingresos tardan en hacerse efectivos les conviene este tipo de créditos, a los prestatarios les favorece ya que se disminuye el riesgo de crédito y de iliquidez, ya que esta modalidad permite cuotas bajas al inicio las cuales se incrementan cada periodo según el gradiente ideal. Esto permite al deudor aprovechar el crédito al máximo hasta fortalecerse, ejemplo muy claro es el sector agrícola o el de la construcción, igualmente se pueden pactar créditos con gradientes en forma decreciente o escalonados según sea el caso; lo ideal es que se diseñen créditos en los que se pueden negociar la tasa, el tiempo y muy en especial el valor del gradiente. El gráfico $\mathrm{N}^{0} 7$ que nos muestra el comportamiento del ejemplo anterior.

\section{Análisis de alternativas de crédito en vista gráfica}

El software nos permite analizar otras alternativas y cambios en las modalidades de pagos, veamos qué pasaría si otro banco que llamaremos B ofreciera la siguiente alternativa, la Compañía XYZ necesita realizar un crédito de $\$ 13.500 .000$ millones de pesos, si debe pagar el crédito en 12 meses a una tasa del $2 \%$, cuál será el valor de cada una de las cuota, si se tiene como condición un gradiente creciente vencido de $\$ 180.000$ pesos cada mes, Las variable y sus valores se presentan en la tabla $\mathrm{N}^{\circ} 4$. 
Gráfico N7. Vista grafica Vista Hoja de cálculo para el cálculo del gradiente creciente

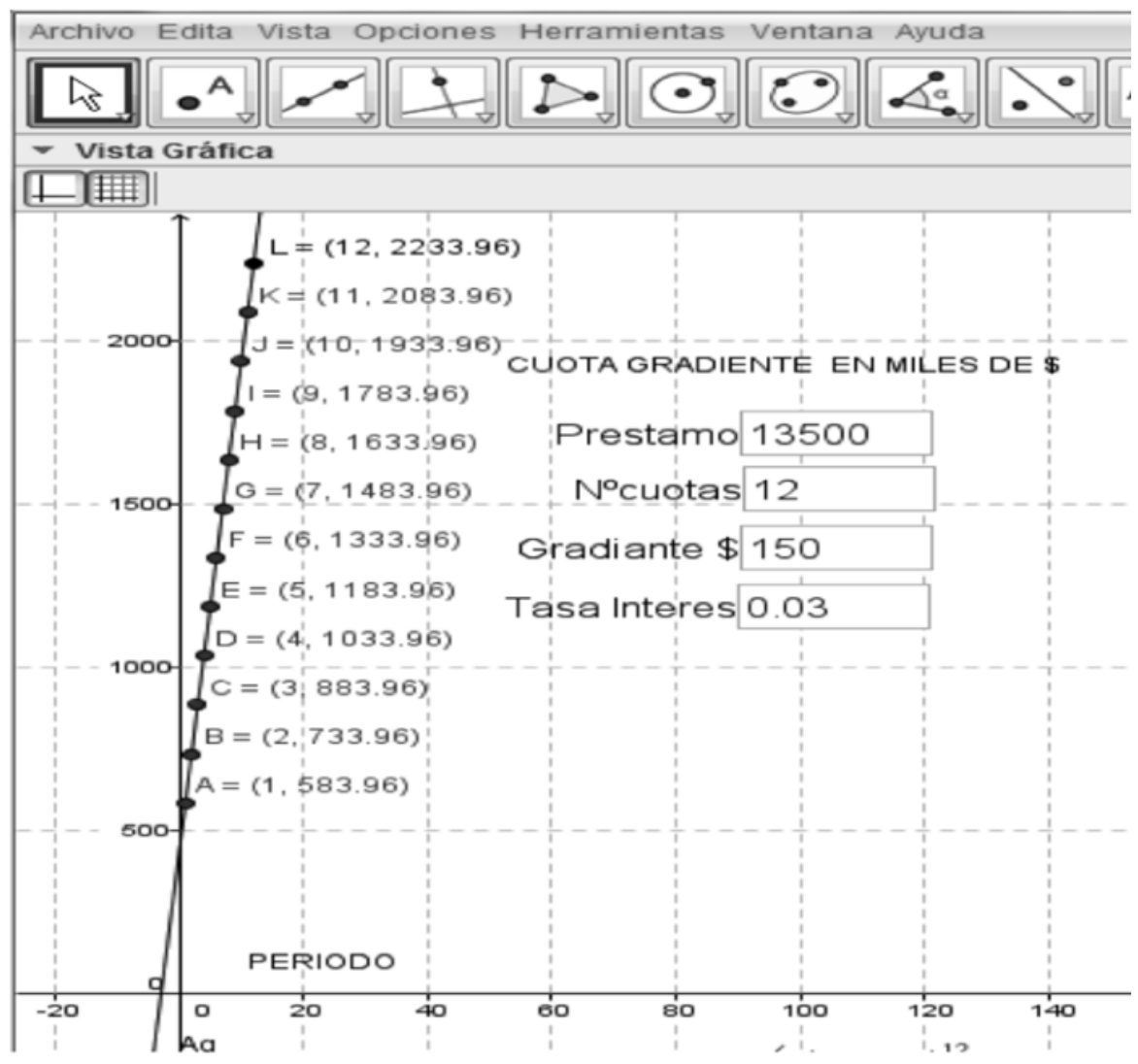

Nota: cifras del préstamo en miles de pesos. Fuente: cálculos del autor con GEOGEBRA.

Tabla $\mathbf{N}^{\circ} 4$. Datos para hallar la cuota de pagos con "Gradiente" creciente.

\begin{tabular}{|l|l|}
\hline $\mathrm{n}=$ tiempo en meses. & $\mathrm{n}=12$ \\
$\mathrm{i}=$ la tasa de interés. & $\mathrm{i}=$ la tasa de interés, $2 \%$ mensual \\
$\mathrm{P}=$ capital solicitado. & $\mathrm{P}=$ capital solicitado $\$ 13.500 .000$ \\
$\mathrm{G}=$ gradiente & $\mathrm{G}=\$ 180.000$ \\
$\mathrm{~A}=$ cuota a pagar & $\mathrm{A}=$ cuota a pagar \\
\hline
\end{tabular}

Fuente: autor con base en Orosco (2008)

No menos importante es el análisis del crédito, el software permite muchas cosas como graficar los intereses, el abono a capital, se pueden analizar cambios al variar la tasa, el tiempo y el valor del gradiente. Lo importante es la posibilidad de observar el comportamiento de la deuda, si la modalidad de pago conviene o no, si es posible pagarlo, controlando el riesgo de iliquidez y de crédito.

Es lógico que el valor de los intereses a pagar cambie notablemente al variar las condiciones de pago, con el banco A se pagan un total de intereses de $\$ 3.407 .559,39$ y con el banco B $\$ 2.327 .891,88$ y si se totalizan el valor del total de cuotas pagadas, Banco A \$16.907.559,39 y con el Banco B $\$ 15.827 .891,88$. Se puede observar que es más adecuado tomar la opción del banco B. También se puede apreciar que con el banco $B$ se inicia pagando cuotas más bajas que en $A$, pero las ultimas cuotas son superiores a la del banco A, lo ideal es calcular los posibles flujos de caja futuros y tomar la decisión más conveniente.

El análisis del comportamiento del crédito se aprecia desde la primera cuota y cómo cambia el valor de cada una de ellas. Es fundamental contar con estas modalidades de pago que hoy por hoy son más que necesarias para una adecuada bancarización. El acceso al crédito requiere del uso de otras formas no tradicionales, este trabajo considera que los sectores colombianos deben proponer cambios a las formas tradicionales de pago que, según la banca el crédito está abierto al $100 \%$ de los usuarios, pero solo llega a 12 de cada 100 pymes (Portafolio, 2013). Se necesita, en consecuencia, un sistema financiero más dinámico para atender a este sector y al de la construcción, la agricultura y a los municipios, los cuales se consideran no aptos para crédito, el gráfico $\mathrm{N}^{\circ} 8$, muestra la nueva propuesta a analizar. 
Gráfico N8. Vista grafica Vista Hoja de cálculo para el cálculo del gradiente creciente

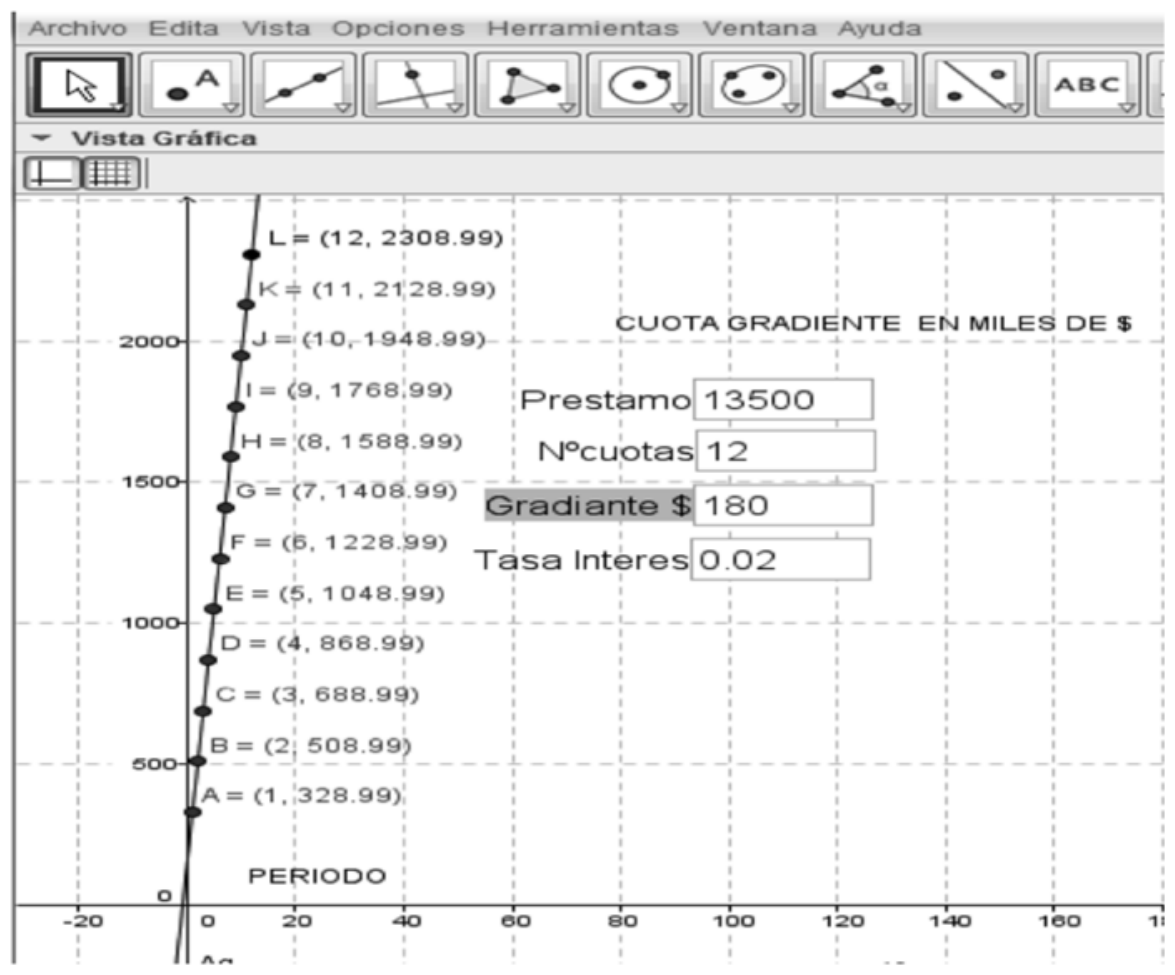

Nota: cifras del préstamo en miles de pesos. Fuente: cálculos del autor con GEÓGEBRA.

La alternativa que ofrece el banco B inicia con una cuota más baja $\$ 328.999$ y termina con una cuota un poco más alta que la propuesta del banco $\mathrm{A}$, en el periodo $\mathrm{N}^{\circ} 12$ de $\$ 2.308 .990$, es importante analizar varias alternativas y propuestas de pago. El deudor podrá analizar cual banco le conviene más y cual no compromete la estabilidad económica, se debe analizar el crédito en términos de capacidad y límite de endeudamiento, deudores $y$ acreedores se deben acercar y negociar mejores condiciones que se adapten a la situación.

La gerencia debe contar con herramientas para analizar si el crédito solicitado es el adecuado para la empresa (Brachfield, 2009). Estas herramientas ayudan a analizar si nivel de deuda es el adecuado y lo más importante si este puede ser pagado, los créditos deben mantenerse en niveles aceptables, de tal suerte que no se afecte la estabilidad económica de la empresa (Viveros, 2013).

Utilizar capital ajeno es apalancarse (Gitman \& Núñez, 2003) $y$, al reducir el capital inicial que es necesario aportar, se produce un aumento de la rentabilidad obtenida. El crédito permite acceder hoy a lo que de otra forma, llevaría meses o años adquirir. En las sociedades modernas prácticamente no hay consumo importante sin crédito, es símbolo de desarrollo social y económico. Así, el crédito para una economía sana es muy importante (Zuñiga, 1987). Aprender acerca del funcionamiento del crédito puede ayudar a las gerencias a reducir costos y evitar utilizar más crédito del adecuado (García, 2003).

Este artículo no pretende mostrar el uso de GEOGEBRA, pero si se considere su uso como apoyo en la comprensión del comportamiento de un crédito, y que se amplié su uso por parte de las empresas y la banca misma. Considera este trabajo que es necesario que ambas partes se ayuden en el otorgamiento del crédito y se diseñen muevas modalidades y formas de pago de crédito.

\section{CONCLUSIONES}

ASOBANCARIA debe apoyar la bancarización en Colombia creando canales y mecanismos de acceso a la financiación para el fondeo de préstamos a sectores no aptos para el sistema financiero colombiano como es el caso del sector agrícola, construcción, exportadores, municipios y pymes, la posibilidad de pago utilizando gradiente permite fortalecer la empresa ya que podrían pagar cuotas de muchas formas creciente, decreciente, escalonada y con múltiples combinaciones, anticipada, vencida, etcétera. 
Los pagos de créditos con gradiente ayudan en la evaluación de las condiciones de acceso al sistema financiero colombiano de sectores considerados por ASOBANCARIA como no aptos para ser sujetos de crédito o financiación. Si bien, el otorgamiento de créditos es potestad directa de las entidades financieras que hacen parte del sistema, el dejar a estos sectores por fuera de las oportunidades para el otorgamiento de cupos y líneas de crédito vulnera en el largo plazo el desarrollo económico. Aplicando nuevas formas de pago se verán favorecidos una lista de sectores presentada por la propia ASOBANCARIA.

Un profesional que gerencia una empresa además de conocer las diferentes modalidades de crédito (Meza, 2009), deberá contar con herramientas que le ayuden a solucionar problemas de una manera rápida, especialmente en lo que concierne a las formas de pago, en razón a que muchas empresas necesitan el crédito bancario (Gómez, 2005). También es importante comprender que las herramientas computacionales ayudan a comprender el comportamiento de este tipo de créditos, lo que apoya la toma de decisiones.

La fórmula de las matemáticas financieras es de fácil aplicación y con la herramienta del software GEOGEBRA se permite una apreciación grafica muy dinámica, la herramienta sirve ya sea para ser aplicada en la empresa como apoyo en la toma de decisiones o como guía de asesoría (INTEF, 2013), su cálculo ayuda de manera inmediata a apreciar gráficamente los efectos por variaciones en las condiciones del crédito.

\section{REFERENCIAS}

Aguirre, H. M. (2008). Matemáticas financieras, México: Cengage Learning Editores.

Brachfield, P. (2009). Gestión del crédito y cobro, una visión del crédito empresarial, Barcelona: Profit Editorial.

Colombia Aprende. (s.f.). Acceso al banco de objetos de aprendizaje. Recuperado el 01 de 05 de 2013, de http://www.colombiaaprende.edu.co/html/directivos/1598/ article-99543.html

Costa, V. A., \& Vacchino, M. C. (2013). Taller VI Visualización de objetos matemáticos con GEOGEBRA para la enseñanza media. Recuperado el 02 de 05 de 2013, de http:// www.jornadasceyn2.fahce.unlp.edu.ar/talleres/talleres-pdf/ Taller\%20V1.pdf

Ferragina, R., Amman, S.; Bifano F.; Cicala R.; González C.; Lupinacci L. (2012). GEOGEBRA entra al aula de matemática. Buenos Aires: Miño y Davila.
García, S. L. (2003). Administración financiera - fundamentos y aplicaciones. Cali: Prensa Moderna Impresores S.A.

GEOGEBRA (2013). (2013). Guía de Referencia Rápida 4.2. Recuperado el 01 de 02 de 2013, de http://www.GEOGEBRA.org/help/GEOGEBRAquickstart_es.pdf

Gitman, L. J., \& Núñez, E. N. (2003). Principios de administración financiera apalancamientos financieros. En L. J. GITMAN. Principios de administración financiera. México: Editorial Pearson.

Gómez, A. R. (2005). Matemáticas financiera. Bogotá: UNAD.

Guzmán, C. A. (2006). Matemáticas financieras para toma de decisiones empresariales. México: Editor Juan Carlos Martínez Coll.

Hohenwarte. M. (2013) GEOGEBRA Versión 4.2.16. Software libre descargado de http://www.GEOGEBRA.org/cms/ es/download/

Hohenwarter, J., \& Hohenwarter, M. (2008). Introduction to GEOGEBRA. En M. Hohenwarter. Florida: FCR-STEM, Learning systems institute, Florida state university.

Horne, J. C., \& Wachowics, J. M. (2002). Fundamentos de administración financiera. México: Pearson Educación.

INTEF. (2013). GEOGEBRAen la enseñanza de Matemáticas. Recuperado el 01 de 05 de 2013, de http://formacionprofesorado.educacion.es/index.php/es/materiales/236-GEOGEBRA-en-la-ensenanza-de-matematicas

José, L. V., \& Valenzuela, P. L. (2001). Matemáticas financieras. México: Pearson Educación.

Matemática (2012). Guía para comenzar con GEOGEBRA. Recuperado el 09 de 04 de 2013 de http://www.profematematica.com.ar/index.php?topic=13.msg33\#msg33

Medina, R. S. (2008). El riesgo de crédito en el marco del acuerdo de Basilea II. Madrid: Delta Publicaciones.

Meza, J. J. (2008). Matemáticas Financieras Aplicadas. Bogotá: ECOE EDICIONES.

Morales, C. M. (2012). Matemáticas financieras, Gradientes. Medellín: Propia, CMM.

Navarro, D. (2013). Series gradientes uniformes geométricas. Tomado de Universidad Nacional de Colombia, Curso ingeniería económica. Recuperado el 03 de 05 de 2013, de http://www.virtual.unal.edu.co/cursos/sedes/manizales/4010045/Lecciones/Cap\%207/SGUG.htm 
Portafolio (2013). Solo el 12 por ciento de las pymes tiene acceso al crédito. Recuperado el 01 de 05 de 2013, de http://www.portafolio.co/negocios/solo-el-12-ciento-laspymes-tiene-acceso-al-credito

Salcedo, J. R. (2002). Matemáticas financieras, progresiones aritméticas y geométricas. Bogotá: Alfaomega.

Serna, R., \& Rojas, G. (2012). Manual didáctico de Matemáticas Financieras. Bogotá: Universidad EAN.
Viveros, M. (2013). Matemática financiera. Estudio de los métodos y técnicas de la Matemática Financiera. Cali: Universidad del Valle.

Zúñiga, L. (1987). Crédito Cooperativo, importancia del sistema financiero y del crédito en la economía del país. San José: EUNED. 